\title{
A New Time-Varying Feedback RISE Control for 2nd-Order Nonlinear MIMO Systems: Theory and Experiments
}

\author{
Hussein Saied ${ }^{a, b^{*}}$, Ahmed Chemori ${ }^{\mathrm{a}}$, Mohamed Bouri ${ }^{\mathrm{c}}$, Maher El Rafei ${ }^{\mathrm{b}}$, Clovis \\ Francis $^{\mathrm{b}}$ and Francois Pierrot ${ }^{\mathrm{a}}$ \\ ${ }^{a}$ LIRMM, University of Montpellier, CNRS, Montpellier, France; \\ ${ }^{\mathrm{b}}$ CRSI, Lebanese University, Beirut, Lebanon; \\ ${ }^{c}$ LSRO-EPFL, Lausanne, Switzerland
}

\section{ARTICLE HISTORY}

Compiled October 18, 2019

\begin{abstract}
This study focuses on the development of a new class of the Robust Integral of the Sign of the Error (RISE) control law adequate for second-order nonlinear multiinput-multi-output systems. A revisit for the original RISE is done by altering some static feedback gains into time-varying nonlinear ones depending on the system states. The proposed controller takes advantage of both RISE control robustness towards uncertainties and the special behavior of nonlinear feedback gains towards time-varied parameters. A Lyaponuv-based stability analysis to prove the semiglobal asymptotic tracking of the proposed new controller is included. In order to validate the relevance of the proposed controller, real-time experimental results are presented and discussed. Experiments have been conducted on a Delta parallel manipulator, in different operating conditions including payload and speed variations.
\end{abstract}

\section{KEYWORDS}

RISE control, nonlinear feedback gains, parallel kinematic manipulators, stability analysis, real-time experiments.

\section{Introduction}

Nonlinear dynamical systems of uncertainties and parameters variation have been studied extensively in the literature. Those systems have been always considered as a challenge possessing special care from scientific people. In fact, most of the realtime industrial applications are counted in the family of highly nonlinear uncertain dynamical systems.

Recently, a novel control mechanism called Robust Integral of the Sign of the Error (RISE) has been developed in Xian, Dawson, Queiroz, \& Chen (2004). RISE feedback law is a continuous control solution dealing with Multi-Input-Multi-Output (MIMO) high-order nonlinear systems. This non-model-based control strategy can guarantee a semi-global asymptotic tracking under limited assumptions on the system uncertainties and time-varying parameters. RISE-based controllers have been applied in different real-time applications thanks to the robustness and disturbances rejection provided by RISE feedback closed-loop architecture. It has been proved experimen-

\footnotetext{
* Corresponding author. Email: hussein.saied@lirmm.fr
} 
tally the high efficiency of RISE controller for disturbance rejection compared to some classical controllers in a directed energy platform (jitter) (Feemster, 2014), an Autonomous Underwater Vehicle (AUV) (Fischer, Hughes, Walters, Schwartz, \& Dixon, 2014), a two-link robot manipulator (Fischer, Kan, Kamalapurkar, \& Dixon, 2014), a nonlinear teleoperation system (kawai, Namerikawa, 2013), and a DC motor drive system taking into account the static friction (Yao, Jiao, \& Ma, 2014). Moreover, some research works have been proposed and experimented in the literature based on the adaptation algorithms for the gains of RISE control or for the system parameters. In (Kamaldin, Chen, Kong, Teo, \& Tan, 2016), a gain-adaptive RISE feedback strategy was implemented on a dual-drive gantry, and the results had shown improvement of the proposed controller against the conventional RISE controller. Model-based adaptive control laws with RISE feedback have been proposed and applied to different platforms such as hydraulic load simulator (Luo, Yao, Chen, Li, \& Xu, 2017; Yao, Deng, \& Jiao, 2017) and a parallel robot Delta (Bennehar, Chemori, Bouri, Jenni, \& Pierrot, 2017). It has been proved experimentally the high performances of such control schemes compared to the standard RISE control.

Because of the powerful robustness and performance acquired by RISE and RISEbased control strategies, the idea of improving such controller arises. Enriching this control law with more nonlinearity may allow it to compensate for more percentage of high nonlinearities abundant extensively in most of the industrial robotized applications.

In the last decades, a serious need for nonlinear control appeared in the area of automatic control engineering with the fast evolution of technology. Indeed, conventional linear control has been used in a wide range of industrial applications providing a good performance. However, its good performance is limited to a small range operation and around the nominal steady state only. At critical operating conditions (for example: high-speed, high-precision applications), linear control may degrade the performance and even lead to instability while nonlinear control can handle the variation in the nonlinear dynamics preserving the stability and the good performance (Khalil, 2002; Slotine \& Li, 1991). One of the most studied concepts in the area of nonlinear control is utilizing nonlinear functions as feedback gains able to adapt itself with the variation of the system states, control inputs or other variables. In (Jingqing, 1994), it has been proposed to improve the classical Proportional-Derivative-Integral (PID) controller to a Nonlinear PID (NLPID) by using tracking-differentiator and a nonlinear combination in order to enhance the adaptability and robustness of such regulator. The nonlinear feedback gains concept of NLPID has been applied later to different experimental platforms showing better control performance compared to the static feedback gains theory. One can mention: a NLPID controller applied to a parallel robot $\mathrm{Su}$, Duan, \& Zheng, 2004), an Augmented NLPD controller applied to a serial manipulator (Kelly \& Carelli, 1996), a parallel robot (Shang, Cong, Li, \& Jiang, 2009), and an underwater vehicle (Campos, Chemori, Creuze, Torres, \& Lozano, 2017), and an extended Desired Compensation Adaptation Law (DCAL) applied to a parallel robot (Bennehar, Chemori, \& Pierrot, 2016).

To this point, we have seen the advantages of using nonlinear feedback algorithms for the control problem of complex robotic systems. Parallel Kinematic Manipulators (PKMs) inherit complexity from their closed-kinematic-chains structure in which the moving platform is linked to the fixed-base by at least two kinematic chains (Merlet, 2006). They are well known with their highly nonlinear and coupled dynamics, abundant uncertainties, parameters variation and actuation redundancy (in some PKMs) increasing more the challenge of the control task. Several control solutions have been 
proposed for PKMs in the literature. One can mention the simple linear PID controller applied in (Chaudhary \& Ohri, 2016) and the improved NLPID tested in (Su et al., 2004). Some controllers that have a priori knowledge about the dynamics of the parallel robot such as: computed-torque control (Codourey, 1998), augmented nonlinear PD (Shang et al., 2009), PD with computed feedforward (Saied, Chemori, El Rafei, Francis, \& Pierrot, 2018), dual-space control (Natal, Chemori, \& Pierrot, 2015). In addition, the dynamic adaptive controllers that can calibrate the system parameters online include: RISE-based adaptive control (Bennehar et al., 2017), DCAL (Bennehar et al., 2016), nonlinear dual-mode adaptive Control (Natal, Chemori, \& Pierrot, 2016), adaptive terminal sliding mode control (Bennehar, El Ghazaly, Chemori, \& Pierrot, 2016), $\mathcal{L} 1$ adaptive control with adaptive feedforward (Bennehar, Chemori, Pierrot, \& Creuze, 2015).

In this paper, we propose to receive advantage of the nonlinear feedback gains in the RISE feedback control solution. We look to increase the robustness of RISE regulator towards disturbances, uncertainties and variation of system nonlinearities depending on the operating point. This can be achieved by replacing some of the static feedback gains in RISE control with nonlinear time-varying feedback gains as function of the system states: position error, velocity error, and the integral of the position error. The stability analysis of the new proposed time-varying feedback RISE controller is established based on a suggested Lyapunov function proving its semiglobal asymptotic tracking. Then, it is implemented and tested experimentally on a non-redundant 3DOFs PKM called Delta robot for different scenarios and operating conditions. The resulting performance of the proposed controller overcomes the standard RISE controller in terms of precision, high-speed motions, robustness, and control efforts at high-dynamic operating conditions.

The rest of this paper is organized as follows. In section 2 , a background on the original RISE controller is presented. In section 3, the proposed contribution to RISE control is introduced. Section 4 is dedicated to the stability analysis of the proposed control solution. The kinematics and dynamics of Delta robot are detailed in section 5 . In section 6, the real-time experimental results are presented and discussed. Section 7 gives the final conclusion and emphasizes some possible perspectives.

\section{Background on RISE-feedback control}

Consider the second order MIMO nonlinear dynamical systems represented as:

$$
M(\boldsymbol{x}, \dot{\boldsymbol{x}}) \ddot{\boldsymbol{x}}+F(\boldsymbol{x}, \dot{\boldsymbol{x}})=\boldsymbol{u}
$$

where $\boldsymbol{x}(t), \dot{\boldsymbol{x}}(t) \in \mathbb{R}^{n}$ denote the system states: position and velocity respectively, and $\ddot{\boldsymbol{x}}(t) \in \mathbb{R}^{n}$ denotes the acceleration, with " $n$ " actuators. Note that $\boldsymbol{x}(t)$ and $\dot{\boldsymbol{x}}(t)$ are measurable states. $\boldsymbol{u}(t) \in \mathbb{R}^{n}$ represents the control input. $M(.) \in \mathbb{R}^{n \times n}$ and $F(.) \in \mathbb{R}^{n}$ are uncertain nonlinear functions. In most of the real-world robotic systems, the mathematical model in (1) is poorly known and usually formulated with some simplifications, non-modelled phenomena and disturbances.

Let the output tracking error be defined as follows:

$$
e_{1}=x_{d}-x
$$

where $\boldsymbol{x}_{d}(t) \in \mathbb{R}^{n}$ is the desired trajectory. In order to achieve an asymptotic tracking 
of a reference trajectory $\boldsymbol{x}_{d}(t)\left(e_{1} \rightarrow 0\right.$ as $\left.t \rightarrow \infty\right)$, the system and the desired signal should have the assumptions below.

Assumption 1. The matrix $M(.) \in \mathbb{R}^{n \times n}$ is a symmetric positive-definite matrix and satisfies $\forall \xi(t) \in \mathbb{R}^{n}$ the following inequality:

$$
\underline{m}\|\xi\|^{2} \leq \xi^{T} M(.) \xi \leq \bar{m}(\boldsymbol{x})\|\xi\|^{2}
$$

with $\underline{m} \in \mathbb{R}$ is a positive constant, and $\bar{m}(\boldsymbol{x}) \in \mathbb{R}$ is a positive non-decreasing function. Notice that ||.|| stands for the classical Euclidean norm.

Assumption 2. If $\boldsymbol{x}(t)$ and $\dot{\boldsymbol{x}}(t) \in \mathcal{L}_{\infty}$ (measurable and bounded), then $F($.$) is$ bounded. Moreover, the first and second partial derivatives of the elements of $M($. and $F($.$) with respect to \boldsymbol{x}$ and $\dot{\boldsymbol{x}}$ exist and are also bounded.

Assumption 3. The chosen reference trajectory $\boldsymbol{x}_{d}(t) \in \mathbb{R}^{n}$ is differentiable till the 4 th order, and its derivatives are bounded.

$$
\boldsymbol{x}_{d}^{(i)}(t) \in \mathcal{L}_{\infty} \quad \text { for } \quad i=0,1, \ldots, 4
$$

To develop the closed-loop error system equation, we need to introduce the auxiliary errors $\boldsymbol{e}_{2}(t), \boldsymbol{r}(t) \in \mathbb{R}^{n}$ as follows:

$$
\begin{gathered}
\boldsymbol{e}_{2}=\dot{\boldsymbol{e}}_{1}+\alpha_{1} \boldsymbol{e}_{1} \\
\boldsymbol{r}=\dot{\boldsymbol{e}}_{2}+\alpha_{2} \boldsymbol{e}_{2}
\end{gathered}
$$

where $\alpha_{1}, \alpha_{2}$ are positive constant design gains added to increase the flexibility of tuning.

After differentiating (5b) with respect to time, multiplying both sides of the obtained equation by $M(\boldsymbol{x}, \dot{\boldsymbol{x}})$, then using the system dynamics (1), we get the equation below:

$$
M(.) \dot{\boldsymbol{r}}=M(.)\left(\dddot{\boldsymbol{x}}_{d}+\alpha_{1} \ddot{\boldsymbol{e}}_{1}+\alpha_{2} \dot{\boldsymbol{e}}_{2}\right)+\dot{M}(.) \ddot{\boldsymbol{x}}+\dot{F}(.)-\dot{\boldsymbol{u}}
$$

By adding and subtracting the two terms $\left(\frac{1}{2} \dot{M}(.) \boldsymbol{r}\right.$ and $\left.\boldsymbol{e}_{2}\right)$ for the right-hand side of the above-obtained equation (6), it can be rewritten as follows:

$$
M(.) \dot{\boldsymbol{r}}=-\frac{1}{2} \dot{M}(.) \boldsymbol{r}-\boldsymbol{e}_{2}-\dot{\boldsymbol{u}}+N(.)
$$

where $N($.$) is defined as:$

$$
N(.) \equiv N(\boldsymbol{x}, \dot{\boldsymbol{x}}, \ddot{\boldsymbol{x}}, t)=M(.)\left(\dddot{\boldsymbol{x}}_{d}+\alpha_{1} \ddot{\boldsymbol{e}}_{1}+\alpha_{2} \dot{\boldsymbol{e}}_{2}\right)+\dot{M}(.)\left(\ddot{\boldsymbol{x}}+\frac{1}{2} \boldsymbol{r}\right)+\boldsymbol{e}_{2}+\dot{F}(.)
$$

Based on the stability analysis introduced in (Xian et al., 2004), RISE control law that can achieve the control objective is defined as follows:

$$
\boldsymbol{u}(t)=\left(k_{s}+1\right) \boldsymbol{e}_{2}(t)-\left(k_{s}+1\right) \boldsymbol{e}_{2}(0)+\int_{0}^{t}\left(k_{s}+1\right) \alpha_{2} \boldsymbol{e}_{2}(\sigma) d \sigma+\int_{0}^{t} \beta \operatorname{sgn}\left(\boldsymbol{e}_{2}(\sigma)\right) d \sigma
$$


where $k_{s}$ and $\beta$ are two positive constant gains, $\operatorname{sgn}($.$) is the standard signum function.$ The integral of signum can hold smooth bounded disturbances for a sufficient condition on the feedback gain. The second term of (9) is used to ensure a zero input signal at time $t_{0}=0$.

Computing the first time derivative of (9) and substituting in (7) leads to the following closed-loop error system equation:

$$
M(.) \dot{\boldsymbol{r}}=-\frac{1}{2} \dot{M}(.) \boldsymbol{r}-\boldsymbol{e}_{2}-\left(k_{s}+1\right) \boldsymbol{r}-\beta \operatorname{sgn}\left(\boldsymbol{e}_{2}\right)+N(.)
$$

Let's now consider the auxiliary function defined by: $N_{d}(t)=N\left(\boldsymbol{x}_{d}, \dot{\boldsymbol{x}}_{d}, \ddot{\boldsymbol{x}}_{d}, t\right)$. Then if added and subtracted to the right-hand side of (10) leads to:

$$
M(.) \dot{\boldsymbol{r}}=-\frac{1}{2} \dot{M}(.) \boldsymbol{r}-\boldsymbol{e}_{2}-\left(k_{s}+1\right) \boldsymbol{r}-\beta \operatorname{sgn}\left(\boldsymbol{e}_{2}\right)+\tilde{N}+N_{d}
$$

where

$$
\tilde{N}(\boldsymbol{x}, \dot{\boldsymbol{x}}, \ddot{\boldsymbol{x}}, t)=N(\boldsymbol{x}, \dot{\boldsymbol{x}}, \ddot{\boldsymbol{x}}, t)-N_{d}(t)
$$

Thanks to Assumptions 1 and 2 of the nonlinear functions $M($.$) and F($.$) , and As-$ sumption 3 required in the desired trajectory, one can deduce that functions $N_{d}(t)$ and $\dot{N}_{d}(t) \in \mathcal{L}_{\infty}$ (i.e. exist and bounded).

Since $N($.) is continuous, one can show that $\tilde{N}($.) can be upper bounded as follows:

$$
\|\tilde{N}\| \leq \rho(\|\boldsymbol{z}\|)\|\boldsymbol{z}\|
$$

where $\boldsymbol{z}(t)=\left[\begin{array}{lll}\boldsymbol{e}_{1} & \boldsymbol{e}_{2} & \boldsymbol{r}\end{array}\right]^{T}$, and $\rho():. \mathbb{R} \geq 0 \rightarrow \mathbb{R} \geq 0$ is a globally invertible nondecreasing function. For the proof of (13), the reader can refer to Lemma 1 in the appendix of (Patre, 2009).

Referring to (Xian et al., 2004), it can be verified that the control law of (9) insures that all the closed-loop system states are bounded and converge to zero

$$
e_{1}^{(i)}(t) \rightarrow 0 \quad \text { as } \quad t \rightarrow \infty, \quad \text { for } \quad i=0,1,2
$$

as long as the control gain $k_{s}$ is chosen large enough relative to the initial conditions of the system, $\alpha_{1}, \alpha_{2}>1 / 2$, and $\beta$ satisfies the following condition:

$$
\beta>\left\|N_{d}(t)\right\|_{\mathcal{L}_{\infty}}+\frac{1}{\alpha_{2}}\left\|\dot{N}_{d}(t)\right\|_{\mathcal{L}_{\infty}}
$$

where $\|.\|_{\mathcal{L}_{\infty}}$ is the $\mathcal{L}_{\infty}$ norm (Khalil, 2002).

\section{Proposed contribution: time-varying feedback RISE controller}

\subsection{General overview on nonlinear feedback gains in control}

Several controllers depending on nonlinear feedback gains have been already reviewed in the introduction, highlighting their superior tracking performance compared to linear fixed feedback gains. 
Consider the simple case of linear PID control in its classical form as follows:

$$
u(t)=k_{p} e(t)+k_{i} \int_{0}^{t} e(\sigma) d \sigma+k_{d} \dot{e}(t)
$$

where $k_{p}, k_{i}, k_{d} \in \mathbb{R}^{+}$are three static feedback gains needed to be tuned to obtain the desired closed-loop performance, and $e(t)$ is the tracking error. Once tuned, this controller is able to keep the desired performance if no changes appear. However, when the operating conditions change (uncertainties, time-varying parameters, unknown payload, etc), the fixed feedback gains will be no more able to keep the desired performance, and sometimes the closed-loop system even loses the stability. The NLPID controller proposed in (Jingqing, 1994) has a similar structure as a linear PID but the constant gains are substituted with time-varying ones as follows:

$$
u(t)=k_{p}(.) e(t)+k_{i}(.) \int_{0}^{t} e(\sigma) d \sigma+k_{d}(.) \dot{e}(t)
$$

where $k_{p}(),. k_{i}(),. k_{d}($.$) are positive nonlinear functions depending on the model states.$ This formulation allows the control to be automatically online adjusted with the system variations during operation. Thus, better performance can be achieved and wider zone for operating conditions.

Our proposition is summarized by taking advantage of the time-varying feedback gains into the linear part of RISE controller improving its robustness and global performance. In the forthcoming sections, we introduce our proposed controller followed by a study of the stability of the resulting closed-loop system.

\subsection{Proposed time-varying feedback RISE controller}

The original controller in (9) can be split up into two parts: a linear feedback part based on the measured combined error $\boldsymbol{e}_{2}$, and a nonlinear signum function. The linear part consists of proportional and integral actions on the combined error, which is similar to a PI controller but taking as input the combined error instead of the position error. These two linear control actions may lead up to poor performances when dealing with highly nonlinear systems at critical dynamic operating conditions. They have considerable sensitivity to disturbances and limited tuning capabilities.

We propose to replace the proportional and the integral static feedback gains by nonlinear time-varying ones. The proposed time-varying feedback RISE controller is given as follows:

$$
\begin{aligned}
\boldsymbol{u}(t)=\left(K_{s}(.)+1\right) \boldsymbol{e}_{2}(t) & -\left(K_{s}\left(t_{0}\right)+1\right) \boldsymbol{e}_{2}(0)+\int_{0}^{t}\left(k_{s 0}+1\right) \alpha_{2}(.) \boldsymbol{e}_{2}(\sigma) d \sigma \\
& +\int_{0}^{t} \beta \operatorname{sgn}\left(\boldsymbol{e}_{2}(\sigma)\right) d \sigma
\end{aligned}
$$

with $K_{s}($.$) and \alpha_{2}($.$) are two nonlinear feedback functions designed as suggested in$ 


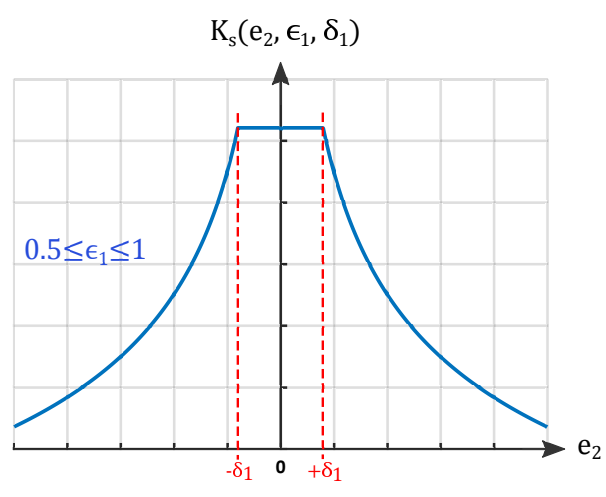

(a) Evolution of $K_{s}\left(e_{2}\right)$.

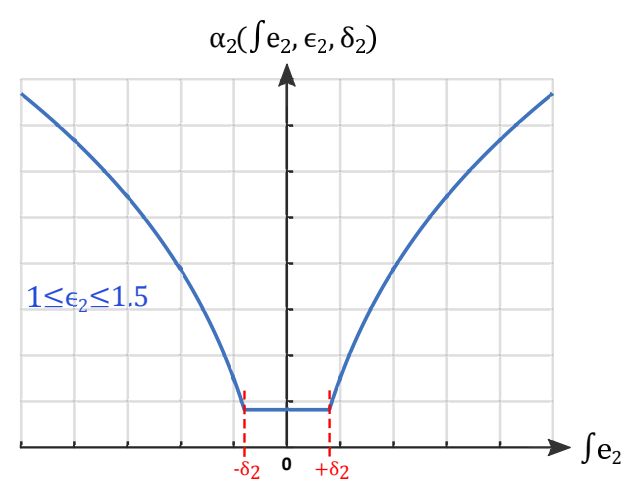

(b) Evolution of $\alpha_{2}\left(\int e_{2}\right)$.

Figure 1. Plots of the evolution of the proposed nonlinear gains with respect to their arguments.

(Shang et al., 2009):

$$
\begin{gathered}
K_{s}(.) \equiv K_{s}\left(\boldsymbol{e}_{2}, \epsilon_{1}, \delta_{1}\right)= \begin{cases}k_{s 0}\left|\boldsymbol{e}_{2}\right|^{\epsilon_{1}-1}, & \left|\boldsymbol{e}_{2}\right|>\delta_{1} \\
k_{s 0} \delta_{1}^{\epsilon_{1}-1}, & \left|\boldsymbol{e}_{2}\right| \leq \delta_{1}\end{cases} \\
\alpha_{2}(.) \equiv \alpha_{2}\left(\boldsymbol{e}_{2}, \epsilon_{2}, \delta_{2}\right)= \begin{cases}\alpha_{20}\left|\int \boldsymbol{e}_{2}\right|^{\epsilon_{2}-1}, & \left|\int \boldsymbol{e}_{2}\right|>\delta_{2} \\
\alpha_{20} \delta_{2}^{\epsilon_{2}-1}, & \left|\int \boldsymbol{e}_{2}\right| \leq \delta_{2}\end{cases}
\end{gathered}
$$

where $k_{s 0}, \alpha_{20}, \epsilon_{1}, \delta_{1}, \epsilon_{2}, \delta_{2}$ are positive design parameters need to be chosen carefully. Indeed, to meet the desired performance, $\epsilon_{1}$ and $\epsilon_{2}$ are chosen within the intervals [0.5, 1] and $[1,1.5]$ respectively.

On one hand, the selection of $\epsilon_{1}$ within the interval $[0.5,1]$ can reduce the proportional gain $K_{s}($.$) at high combined error values and increase it at small ones (see Figure$ 1-a). As long as the combined error remains within the small interval $\left[-\delta_{1},+\delta_{1}\right]$ around zero, the proportional gain remains constant as a maximum saturated value. Notice that the combined error gives knowledge about both position and velocity errors. Thus, such variation of the gain could result in a rapid transition of the closed-loop system states and favorable damping.

On the other hand, the nonlinear feedback gain $\alpha_{2}($.$) varies as function of the$ integral of the combined error (see Figure 1-b), which means that it is more concerned with the steady state combined errors (i.e. errors that persist with time). The choice of $\epsilon_{2}$ within the interval $[1,1.5]$ gives large integral gain for the large steady state combined errors, and small integral gain for the small steady state combined errors as illustrated in Figure 1-b. As long as this error remains within the small interval $\left[-\delta_{2},+\delta_{2}\right]$ around zero, the integral gain remains as a minimum constant value. This variation may accelerate the tracking process towards the setpoint and prevent the integral term from accumulating above or below specific bounds which can solve the integral windup problem.

Choosing $\epsilon_{1}$ and $\epsilon_{2}$ in their corresponding intervals leads to globally bounded nonlinear functions as follows (bounds can be realized from Figure 1):

$$
0<K_{s m} \triangleq k_{s 0}\left\|\boldsymbol{e}_{2}\right\|_{\infty}^{\epsilon_{1}-1} \leq K_{s}(.) \leq k_{s 0} \delta_{1}^{\epsilon_{1}-1} \triangleq K_{s M}
$$




$$
0<\alpha_{2 m} \triangleq \alpha_{20} \delta_{2}^{\epsilon_{2}-1} \leq \alpha_{2}(.) \leq \alpha_{20}\left\|\int \boldsymbol{e}_{2}\right\|_{\infty}^{\epsilon_{2}-1} \triangleq \alpha_{2 M}
$$

where $\|.\|_{\infty}$ indicates the infinity-norm.

Using the above introduced time-varying feedback gains in the standard equation of RISE controller may enhance the global tracking performance of such controller and may improve its robustness towards changes in system parameters. It is worth to confirm that the structure of the nonlinear functions is simple enough to be implemented in real-time experiments.

In order to analyse the stability of the proposed time-varying feedback RISE controller, we need to establish its related closed-loop error equation based on the nonlinear MIMO system (1).

Let us first define the auxiliary error $r(t)$ which is synthesized now using the nonlinear function $\alpha_{2}($.$) as follows:$

$$
\boldsymbol{r}=\dot{\boldsymbol{e}}_{2}+\alpha_{2}(.) \boldsymbol{e}_{2}
$$

Following the same previous procedure: differentiating $r(t)$, multiplying both sides by $\mathrm{M}($.$) , getting use of the system dynamics (1), and arranging the elements of the$ obtained equation leads to:

$$
M(.) \dot{\boldsymbol{r}}=-\frac{1}{2} \dot{M}(.) \boldsymbol{r}-\boldsymbol{e}_{2}-\dot{\boldsymbol{u}}+N(.)
$$

where $N($.$) is a new auxiliary function defined as follows:$

$$
\begin{gathered}
N(.) \equiv N(\boldsymbol{x}, \dot{\boldsymbol{x}}, \ddot{\boldsymbol{x}}, t)=M(.)\left(\dddot{\boldsymbol{x}}_{d}+\alpha_{1} \ddot{\boldsymbol{e}}_{1}+\alpha_{2}(.) \dot{\boldsymbol{e}}_{2}+\dot{\alpha}_{2}(.) \boldsymbol{e}_{2}\right) \\
+\dot{M}(.)\left(\ddot{\boldsymbol{x}}+\frac{1}{2} \boldsymbol{r}\right)+\boldsymbol{e}_{2}+\dot{F}(.)
\end{gathered}
$$

The equation of the closed-loop error system is then derived by differentiating the control law of time-varying feedback RISE controller (18) with respect to time and substituting it in (22). Introducing the supplementary function $\tilde{N}(., t)$ as in (12) allows the closed-loop error equation to be as follows:

$$
\begin{gathered}
M(.) \dot{\boldsymbol{r}}=-\frac{1}{2} \dot{M}(.) \boldsymbol{r}-\boldsymbol{e}_{2}-\dot{K}_{s}(.) \boldsymbol{e}_{2}-\left(K_{s}(.)+1\right) \dot{\boldsymbol{e}}_{2}-\left(k_{s 0}+1\right) \alpha_{2}(.) \boldsymbol{e}_{2} \\
-\beta \operatorname{sgn}\left(\boldsymbol{e}_{2}\right)+\tilde{N}+N_{d}
\end{gathered}
$$

Since $\alpha_{2}($.$) is continuous, the upper bound of \|\tilde{N}\|$ in (13) still exist.

\subsection{Stability analysis of the proposed time-varying feedback RISE controller}

Theorem 3.1. The control law proposed in (18) applied to the second-order nonlinear MIMO system whose dynamic model is governed by (1) ensures that all the system signals are bounded and converge asymptotically to zero with time going to infinity, knowing that the design control gains are chosen such that

$$
\beta>\left\|N_{d}(t)\right\|_{\mathcal{L}_{\infty}}+\left(1 / \alpha_{2 M}\right)\left\|\dot{N}_{d}(t)\right\|_{\mathcal{L}_{\infty}}
$$


with $\alpha_{1}>1 / 2, \epsilon_{1} \in[0.5,1], \epsilon_{2} \in[1,1.5]$, and the bounds $K_{s M}, \alpha_{2 m}$ in (20a) and (20b) are chosen large enough.

Proof. Let us first consider the function $L(t) \in \mathbb{R}$ defined as follows:

$$
L(t)=r\left(N_{d}(t)-\beta \operatorname{sgn}\left(\boldsymbol{e}_{2}\right)\right)
$$

With the use of Lemma 1 in (Xian et al., 2004), we can conclude that if $\beta$ is chosen satisfying the following condition:

$$
\beta>\left\|N_{d}(t)\right\|_{\mathcal{L}_{\infty}}+\frac{1}{\alpha_{2 M}}\left\|\dot{N}_{d}(t)\right\|_{\mathcal{L}_{\infty}}
$$

then the following inequality holds:

$$
\int_{0}^{t} L(\tau) d \tau \leq \beta\left|e_{2}(0)\right|-e_{2}(0) N_{d}(0)
$$

Then, an additional function $P(t) \in \mathbb{R}$ needs to be defined as follows:

$$
P(t)=\beta\left|\boldsymbol{e}_{2}(0)\right|-\boldsymbol{e}_{2}(0) N_{d}(0)-\int_{0}^{t} L(\tau) d \tau
$$

knowing that $P(t) \geq 0, \forall t \geq 0$ is ensured from (26) and (27).

We now introduce a continuous differentiable definite positive function $V: \mathbb{R}^{3 n+1} \times$ $\mathbb{R}_{\geq 0} \rightarrow \mathbb{R}_{\geq 0}$ as follows:

$$
V(\boldsymbol{y}, t)=\frac{1}{2} \boldsymbol{e}_{1}^{T} \boldsymbol{e}_{1}+\frac{1}{2} \boldsymbol{r}^{T} M(.) \boldsymbol{r}+P
$$

where $\boldsymbol{y}=\left[\boldsymbol{z}^{T} \sqrt{P}\right]^{T}$ and $\boldsymbol{z}(t)$ is defined above. In view of the characteristics of the matrix $M($.$) stated by Assumption 1$ and its bounds in (3), $V(\boldsymbol{y}, t)$ can be bounded as follows:

$$
\lambda_{1}\|\boldsymbol{y}\|^{2} \leq V(\boldsymbol{y}, t) \leq \lambda_{2}(\|\boldsymbol{y}\|)\|\boldsymbol{y}\|^{2}
$$

being $\lambda_{1}=(1 / 2) \min \{1, \underline{m}\}$ and $\lambda_{2}=(1 / 2) \max \{\bar{m}(\|\boldsymbol{y}\|), 1\}$.

Applying the time derivative of (29), and using equations (24), (25) and (28) leads to:

$$
\begin{aligned}
\dot{V}= & \boldsymbol{e}_{1}^{T} \boldsymbol{e}_{2}-\alpha_{1} \boldsymbol{e}_{1}^{T} \boldsymbol{e}_{1}-\boldsymbol{r}^{T} \boldsymbol{e}_{2}-\dot{K}_{s}(.) \boldsymbol{r}^{T} \boldsymbol{e}_{2}-\left(K_{s}(.)+1\right) \boldsymbol{r}^{T} \boldsymbol{r} \\
& +\left(K_{s}(.)+1\right) \alpha_{2}(.) \boldsymbol{r}^{T} \boldsymbol{e}_{2}-\left(k_{s 0}+1\right) \alpha_{2}(.) \boldsymbol{r}^{T} \boldsymbol{e}_{2}+\boldsymbol{r}^{T} \tilde{N}
\end{aligned}
$$

where $\dot{K}_{s}($.$) is the time derivative of the nonlinear function K_{s}($.$) . Now, we need to$ find an upper bound for $\dot{V}$ in (31).

Using the conventional inequality for any two vectors, $a$ and $b$ namely $a^{T} b \leq\left(\|a\|^{2}+\right.$ 
$\left.\|b\|^{2}\right) / 2$, one can write:

$$
\begin{aligned}
\dot{V} \leq & \frac{1}{2}\left\|\boldsymbol{e}_{1}\right\|^{2}+\frac{1}{2}\left\|\boldsymbol{e}_{2}\right\|^{2}-\alpha_{1}\left\|\boldsymbol{e}_{1}\right\|^{2}-\frac{1}{2}\|\boldsymbol{r}\|^{2}-\left\|\boldsymbol{e}_{2}\right\|^{2}-\frac{\left|K_{s m p}\right|}{2}\|\boldsymbol{r}\|^{2} \\
& -\frac{\left|K_{s m p}\right|}{2}\left\|\boldsymbol{e}_{2}\right\|^{2}-\left(K_{s m}+1\right)\|\boldsymbol{r}\|^{2}+\frac{\left(K_{s M}+1\right) \alpha_{2 m}}{2}\|\boldsymbol{r}\|^{2} \\
& +\frac{\left(K_{s M}+1\right) \alpha_{2 m}}{2}\left\|\boldsymbol{e}_{2}\right\|^{2}-\frac{\left(k_{s 0}+1\right) \alpha_{2 m}}{2}\|\boldsymbol{r}\|^{2}-\frac{\left(k_{s 0}+1\right) \alpha_{2 m}}{2}\left\|\boldsymbol{e}_{2}\right\|^{2} \\
& +\|\boldsymbol{r}\| \rho(\|\boldsymbol{z}\|)\|\boldsymbol{z}\|
\end{aligned}
$$

where $K_{s m p}$ is a lower bound for $\dot{K}_{s}($.$) . After developing and re-arranging (32) we$ obtain:

$$
\dot{V} \leq-\zeta_{1}\left\|\boldsymbol{e}_{1}\right\|^{2}-\zeta_{2}\left\|\boldsymbol{e}_{2}\right\|^{2}-\zeta_{3}\|\boldsymbol{r}\|^{2}-\mu\|\boldsymbol{r}\|^{2}+\|\boldsymbol{r}\| \rho(\|\boldsymbol{z}\|)\|\boldsymbol{z}\|
$$

where $\zeta_{1}, \zeta_{2}, \zeta_{3}$ and $\mu$ are constants to be chosen positive defined as follows:

$$
\begin{gathered}
\zeta_{1}=\alpha_{1}-\frac{1}{2} \\
\zeta_{2}=\frac{1}{2}\left(1-K_{s M} \alpha_{2 m}+k_{s 0} \alpha_{2 m}\right) \\
\zeta_{3}=\frac{1}{2}\left(3+2 K_{s m}-K_{s M} \alpha_{2 m}-\alpha_{2 m}\right) \\
\mu=\frac{1}{2}\left(k_{s 0}+1\right) \alpha_{2 m}
\end{gathered}
$$

From (34a), $\alpha_{1}$ should satisfy the condition $\alpha_{1}>1 / 2$. Equation (33) can be rewritten as follows:

$$
\dot{V} \leq-\lambda_{3}\|\boldsymbol{z}\|^{2}-\left(\mu\|\boldsymbol{r}\|^{2}-\|\boldsymbol{r}\| \rho(\|\boldsymbol{z}\|)\|\boldsymbol{z}\|\right)
$$

being $\lambda_{3}=\min \left\{\zeta_{1}, \zeta_{2}, \zeta_{3}\right\}$. Using the mathematical remarkable square identities, (35) can be rewritten as follows:

$$
\dot{V} \leq-\left(\lambda_{3}-\frac{\rho^{2}(\|\boldsymbol{z}\|)}{4 \mu}\right)\|\boldsymbol{z}\|^{2} \triangleq-c\|\boldsymbol{z}\|^{2}
$$

where $c$ is some positive constant, which implies that the following inequality holds:

$$
\lambda_{3}>\frac{1}{4 \mu} \rho^{2}(\|\boldsymbol{z}\|)
$$


Let us define the region $\mathcal{D}$ using inequality (37) as follows:

$$
\mathcal{D}=\left\{\boldsymbol{y} \in \mathbb{R}^{3} \times \mathbb{R}_{\geq 0} \mid \quad\|\boldsymbol{y}\|<\rho^{-1}\left(2 \sqrt{\lambda_{3} \mu}\right)\right\}
$$

We know that $V(\boldsymbol{y}, t) \in \mathcal{L}_{\infty}$ is a continuously differentiable function such that $W_{1}(\boldsymbol{y}) \leq V(\boldsymbol{y}, t) \leq W_{2}(\boldsymbol{y})$ (see equation $\left.(30)\right)$ and $\dot{V}(\boldsymbol{y}, t) \leq-W(\boldsymbol{y})$ (from equation (36)). Hence $\boldsymbol{e}_{1}, \boldsymbol{e}_{2}, \boldsymbol{r} \in \mathcal{L}_{\infty}$.

$W_{1}(\boldsymbol{y}), W_{2}(\boldsymbol{y})$ are continuous positive-definite functions $\forall t \geq 0$ and $\forall \boldsymbol{y} \in \mathcal{D}$, and $W(\boldsymbol{y})$ is uniformly continuous positive-semidefinite function.

Given that the initial conditions $\boldsymbol{y}(0) \in \mathcal{S}$, a subset of $\mathcal{D}$ defined as follows:

$$
\mathcal{S}=\left\{\boldsymbol{y} \subset \mathcal{D} \mid \quad W_{2}(\boldsymbol{y})<\lambda_{1}\left(\rho^{-1}\left(2 \sqrt{\lambda_{3} \mu}\right)\right)^{2}\right\}
$$

then we can conclude, using Lemma 2 of (Xian et al., 2004), that $\|\boldsymbol{z}(t)\|^{2} \rightarrow 0$ as $t \rightarrow \infty, \forall \boldsymbol{y}(0) \in \mathcal{S}$. This means that all the closed-loop system states $\left(\boldsymbol{e}_{1}, \boldsymbol{e}_{2}, \boldsymbol{r}\right)$ asymptotically converge to zero with time.

$$
\boldsymbol{e}_{1}^{(i)}(t) \rightarrow 0 \quad \text { as } \quad t \rightarrow \infty \quad \forall \boldsymbol{y}(0) \in \mathcal{S}
$$

and here the proof is concluded.

\section{Control application to Delta parallel robot}

\subsection{Description and modeling of Delta parallel robot}

Delta parallel robot is a 3-DOF non-redundant PKM that has been patented by prof. Reymond Clavel (Clavel, 1990) and developed at Ecole Polytechnique Federale de Lausanne (EPFL), Switzerland. It has been proposed as an industrial robot for highspeed pick-and-place applications.

The robot includes a fixed-base support and a moving platform (traveling-plate) linked through three assemblies. Each assembly is a kinematic chain formed of a movable rear-arm mounted from its first extremity to the shaft of a motor supposed to make rotation, and linked from the second extremity to two parallel bars, which compose the forearm, by means of ball-and-socket passive joints. The parallel rods are mounted to the traveling-plate from their other side using the same said joints. This structure enables the control of three basic degrees of freedom $(x, y$ and $z)$ with preserving the parallelism of the traveling-plate with respect to the fixed-base. The inclination and orientation of the moving platform in space remain unchanged whatever the motion of the moving parts of the robot. Figure 2 illustrates the kinematics of Delta parallel robot.

The 3-dimensional coordinate vector $X=\left[\begin{array}{lll}x & y & z\end{array}\right]^{T}$ represents the position of center of mass of the traveling-plate in the workspace. For each Cartesian position of the traveling-plate in the workspace, there exist a unique and straightforward inverse kinematic solution of the joint angles in a 3-dimensional coordinate vector $q=\left[\begin{array}{lll}q_{1} & q_{2} & q_{3}\end{array}\right]^{T}$.

The dynamic model of Delta robot is established, as in (Bennehar et al., 2017), based on the virtual work principle. One can find more details on the dynamics of such robot in (Codourey, 1996; Miller \& Boris, 1995). The inverse dynamic equation 


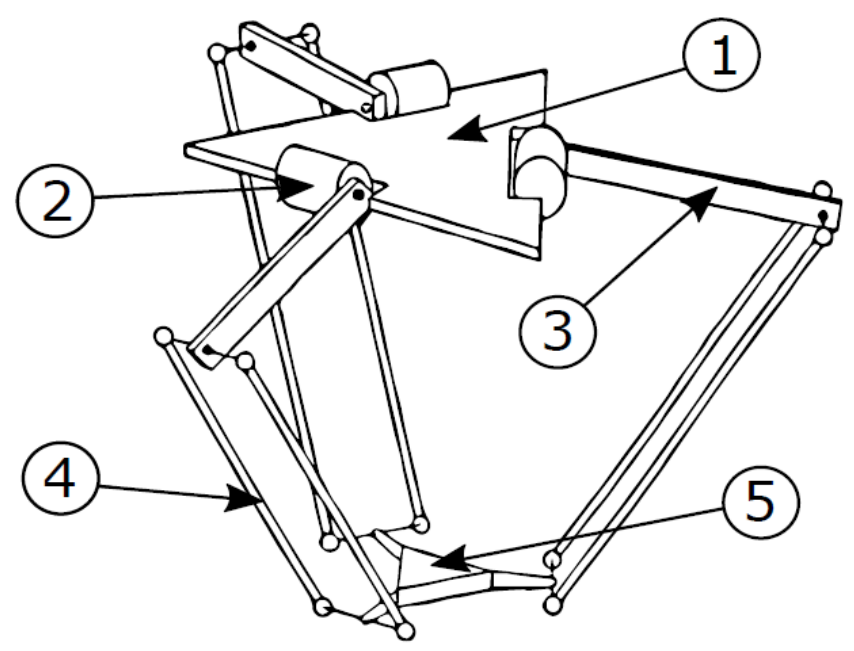

Figure 2. A schematic view of Delta parallel robot including (1): Fixed-base, (2): Actuator, (3): Rear-arm, (4): Forearm, (5): traveling-plate.

of Delta robot can be written in joint space as follows:

$$
\Gamma(t)=M(q) \ddot{q}+C(q, \dot{q}) \dot{q}+G(q)
$$

where

- $q, \dot{q}, \ddot{q}$, are the joint position, velocity, and acceleration vectors respectively.

- $M(q)$, is the total mass and inertia matrix of the robot,

- $C(q, \dot{q})$, is the Coriolis and centrifugal forces matrix,

- $G(q)$, is the gravitational forces vector,

- $\Gamma(t)$, is the control input vector.

The main dynamic parameters of delta parallel robot are summarized in Table 1.

\subsection{Application of the proposed controller to Delta robot}

In order to demonstrate the effectiveness of the proposed time-varying feedback RISE controller, both the original RISE and the proposed control algorithms were implemented on the 3-DOF Delta parallel robot. A comparative study between the two implemented controllers is done in the forthcoming experimental section.

For an adequate control design and implementation, we re-defined the position error in (2) for Delta robot as a difference between the desired joint angle $q_{d} \in \mathbb{R}^{3}$ and the

Table 1. The main dynamic parameters of Delta parallel robot.

\begin{tabular}{cccc}
\hline Parameter & Value & Parameter & Value \\
\hline Rear-arm length: & $240 \mathrm{~mm}$ & Rear-arm mass: & $0.22 \mathrm{~kg}$ \\
Forearm length: & $480 \mathrm{~mm}$ & Forearm mass: & $0.084 \mathrm{~kg}$ \\
Actuator inertia: & $1.82 \times 10^{-3} \mathrm{~kg} \cdot \mathrm{m}^{2}$ & Traveling-plate mass: & $0.305 \mathrm{~kg}$ \\
\hline
\end{tabular}


actual measured one $q \in \mathbb{R}^{3}$ as follows:

$$
e_{1}=q_{d}-q
$$

The measurement of the actual angle position is performed by means of encoders integrated in the motors, and the position in Cartesian space is computed using the forward kinematics of the robot as common for most parallel robots.

The dynamic model of Delta robot (41) is considered as a second order nonlinear MIMO system with a structure similar to the system equation (1) with $n=3$ (the total number of degrees of freedom).

Consequently, the mass and inertia matrix $M(q)$ is a diagonal symmetric positivedefinite matrix satisfying the boundedness condition introduced in Assumption 1. The dynamics of Delta parallel robot satisfy Assumption 2 such that $q(t)$ and $\dot{q}(t)$ are measurable and bounded giving that $C(q, \dot{q})$ and $G(q)$ are bounded. Then, the first and second partial derivatives of $M(q)$ with respect to $q$ and those of $C(q, \dot{q}), G(q)$ with respect to $q, \dot{q}$ exist and bounded. Also, the chosen desired trajectory $q_{d}(t)$ satisfies the assumption of differentiability and boundedness reported in Assumption 3.

Therefore, Delta robot dynamics fit the design of RISE-based controllers and it is possible to implement both control schemes in real-time experiments. The proposed time-varying feedback RISE control architecture is summarized in the block diagram depicted in Figure 3.

\section{Real-time experiments and results}

\subsection{Experimental testbed and implementation issues}

The Delta parallel robot used for the real-time experiments is shown in Figure 4, it is located at Robotics Systems Laboratory, EPFL, Switzerland. Three direct-drive motors integrated with the fixed-base allow the motion of the kinematic chains generating three translational movements of the traveling-plate in $x, y$ and $z$ axes. Each motor can

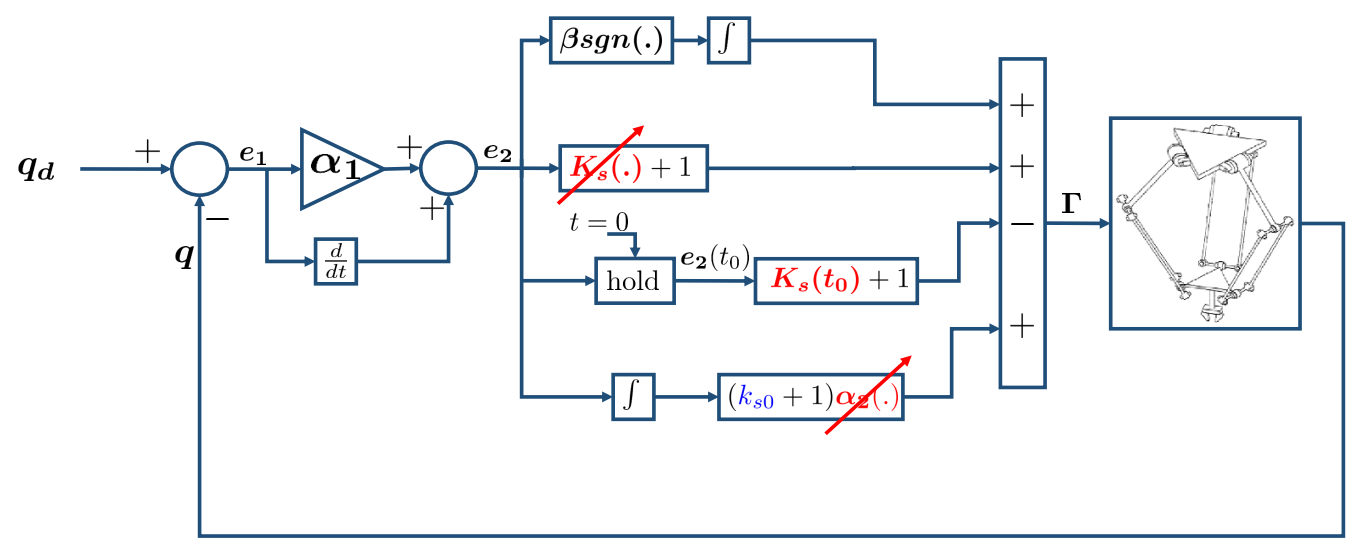

Figure 3. Block diagram of the proposed time-varying feedback RISE control scheme applied to Delta robot in joint space, $K_{s}($.$) and \alpha_{2}($.$) represent the nonlinear feedback gains.$ 


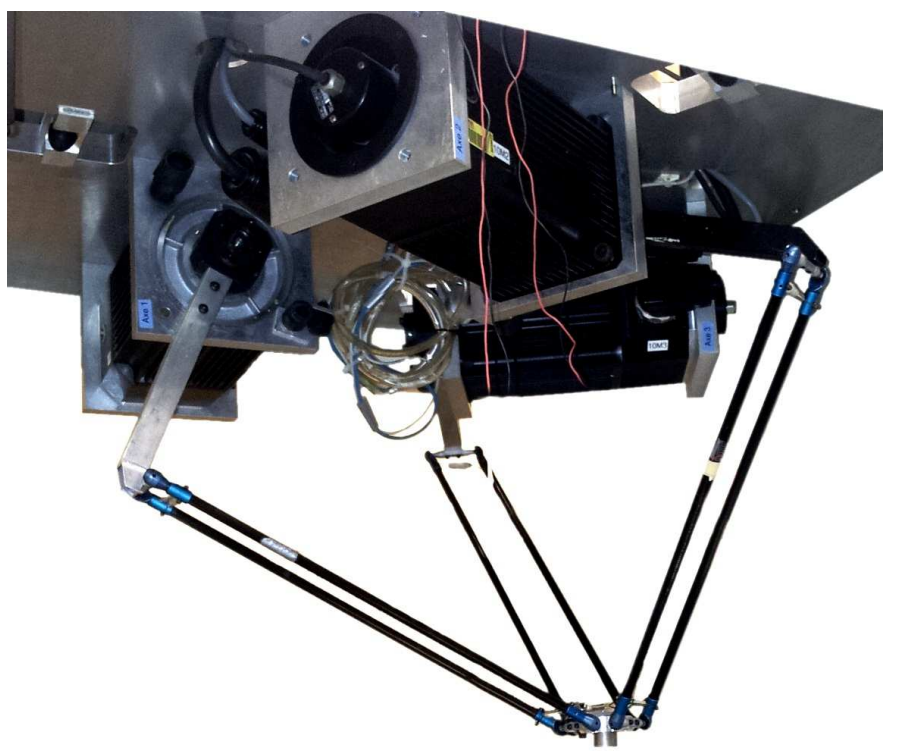

Figure 4. View of the real Delta parallel robot used for real-time experiments.

deliver a maximum torque of $23 \mathrm{Nm}$. The control algorithms are implemented in $\mathrm{C}++$ language level using Visual Studio software from Microsoft, running on a Windows XP operating system. RTX extension is used to establish the real-time communication. The internal timer (HAL timer) of RTX is configured to $100 \mu s$ in which the control loop is set to 10 times this value for synchronization, leading to a sample time $1 \mathrm{~ms}$, and a sampling frequency of $1 \mathrm{KHz}$.

The reference trajectory is generated using semi-ellipse geometric motions producing a pick-and-place trajectory in Cartesian space. This trajectory is mainly used in industry for food packaging applications. A 3D illustrative view is shown in Figure 5 for the pick-and-place cycle to be followed by the robot's traveling-plate, while Figure 6 displays the equivalent desired Cartesian trajectories $x, y$ and $z$ versus time.

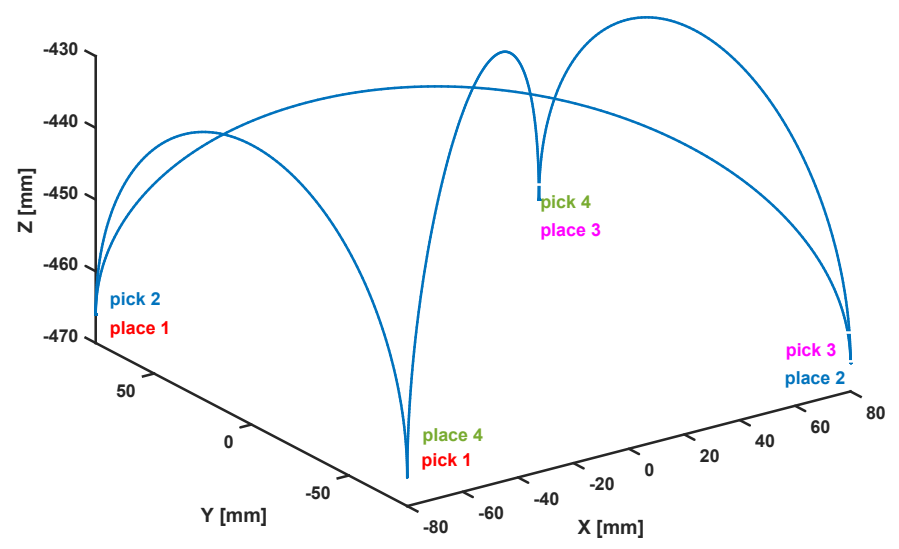

Figure 5. 3D view of pick-and-place reference trajectory in Cartesian space. 

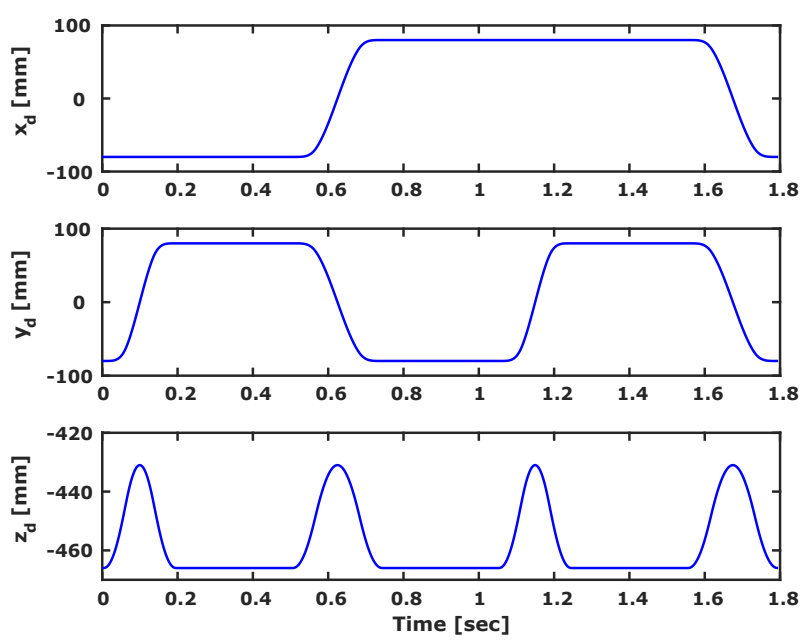

Figure 6. The desired evolution of the three degrees of freedom to be tracked by the robot's traveling-plate.

\subsection{Performance evaluation criteria}

In order to quantify the relevance of the control algorithm, we need to define a certain performance index. One of our main objectives is to enhance the precision and increase the tracking accuracy of Delta robot through the proposed controller.

An accuracy evaluation tool frequently used to evaluate differences between a desired trajectory and a measured one is the Root-Mean-Square Error (RMSE) criterion. It can quantify approximately the error between the desired trajectory and the actual one traversed by the robot.

Two performance indices are used in our case, the RMSEs in Cartesian and joint spaces defined as follows:

$$
\begin{aligned}
R M S E_{x} & =\sqrt{\frac{1}{N} \sum_{i=1}^{N}\left(e_{x}^{2}(i)+e_{y}^{2}(i)+e_{z}^{2}(i)\right)} \\
R M S E_{J} & =\sqrt{\frac{1}{N} \sum_{i=1}^{N}\left(e_{q_{1}}^{2}(i)+e_{q_{2}}^{2}(i)+e_{q_{3}}^{2}(i)\right)}
\end{aligned}
$$

where $e_{x}, e_{y}, e_{z}$ represent the Cartesian position tracking errors along $x, y$, and $z$ axes respectively. $e_{q_{1}}, e_{q_{2}}, e_{q_{3}}$ denote the joints tracking errors. $\mathrm{N}$ is the number of the collected samples through the whole trajectory.

In order to estimate the energy consumption for each controller at high dynamic operating conditions, the input-torques-based criterion is proposed as follows:

$$
E_{\Gamma}=\sum_{i=1}^{3} \sum_{j=1}^{N}\left|\Gamma_{i}(j)\right|
$$

where $E_{\Gamma}$ is the total summation of the absolute value of the input torques delivered by the three actuators. 


\subsection{Tuning of the control gains}

A popular method for tuning of the control gains in experiments, used for complex robotic systems, is the Trial and Error method. It is characterized by trying manually and continuously different sets of control gains in real-time framework until the desired control performance is achieved. It is used mostly when the formulated dynamic model does not exactly match the physical system, and thus the automatic numerical closedloop tuning methods may give unsuitable control gains for real-time experiments.

\subsubsection{Tuning of the standard RISE control gains}

The tuning process of the standard RISE control gains is performed by the following simple procedure:

(1) Set $\alpha_{2}=0$ and $\beta=0$,

(2) tune $\alpha_{1}$ and $k_{s}$ as if dealing with a PD controller, given that $\alpha_{1}\left(k_{s}+1\right)$ is the proportional gain and $\left(k_{s}+1\right)$ is the derivative one till a satisfied tracking is reached,

(3) start increasing $\alpha_{2}$ with modifying again $\alpha_{1}$ and $k_{s}$ either increasing or decreasing till we reach as best performance index as possible,

(4) increase $\beta$ until obtaining less chattering input signal and better performance index.

Following the above procedure, the standard RISE control gains were tuned in realtime experiments, and the obtained final values are summarized in Table 2.

\subsubsection{Tuning of the proposed time-varying feedback RISE control gains}

For the tuning process of the proposed time-varying feedback RISE controller, and especially tuning the nonlinear feedback gains, a similar manner for the one proposed in (Shang et al., 2009) to tune the NPD control gains is used in our case.

The main steps of this procedure are as follows:

(1) Initialization: $\epsilon_{1}=1, \epsilon_{2}=1, \alpha_{20}=0$ and $\beta=0$,

(2) increase $\alpha_{1}$ and $k_{s 0}$ starting both from zero until obtaining acceptable tracking performance.

(3) increase the value of $\alpha_{20}$ to get a better tracking performance, then make a trade-off between $\alpha_{1}, k_{s 0}$ and $\alpha_{20}$,

(4) find $\left(e_{2}\right)_{\max }$ and $\left(\int e_{2}\right)_{\max }$ values and set their halves as values of $\delta_{1}$ and $\delta_{2}$ respectively,

(5) decrease the value of $\epsilon_{1}$ within the interval $[0.5,1]$ and increase the value of $\epsilon_{2}$ within the interval $[1,1.5]$, retune again the values of $k_{s 0}$ and $\alpha_{20}$ making a compromise among the four values,

(6) repeat steps (4) and (5) until obtaining the best possible RMSE,

(7) increase $\beta$ until obtaining better performance index.

Based on the above tuning algorithm, the control parameters of the proposed timevarying feedback RISE controller were tuned experimentally, and the obtained final values are summarized in Table 2. 
Table 2. Summary of the control gains of both controllers tuned by the proposed process.

\begin{tabular}{ccc}
\hline Standard RISE & \multicolumn{2}{c}{ Proposed time-varying feedback RISE } \\
\hline$\alpha_{1}=360$ & $\alpha_{1}=450$ & $\beta=2$ \\
$k_{s}=0.35$ & $k_{s 0}=0.35$ & $\alpha_{20}=0.66$ \\
$\alpha_{2}=0.66$ & $\epsilon_{1}=0.65$ & $\epsilon_{2}=1.45$ \\
$\beta=1.5$ & $\delta_{1}=0.05$ & $\delta_{2}=0.12$ \\
\hline
\end{tabular}

\subsection{Obtained experimental results}

two main scenarios have been experimented with both RISE and time-varying feedback RISE controllers: (i) scenario 1: nominal case and (ii) scenario 2: robustness towards speed and payload changes.

\subsection{Scenario 1: nominal case}

In this scenario, the traveling-plate does not carry additional payload and the Delta robot is operating at acceleration of $2.5 \mathrm{G}$ (with a speed of $1500 \mathrm{~mm} / \mathrm{s}$ ).

Following the reference trajectory shown in Figures 5 and 6 , the Cartesian and joint tracking errors for both controllers are registered and plotted in Figure 7. We can notice that replacing the static feedback gains in the standard RISE controller with
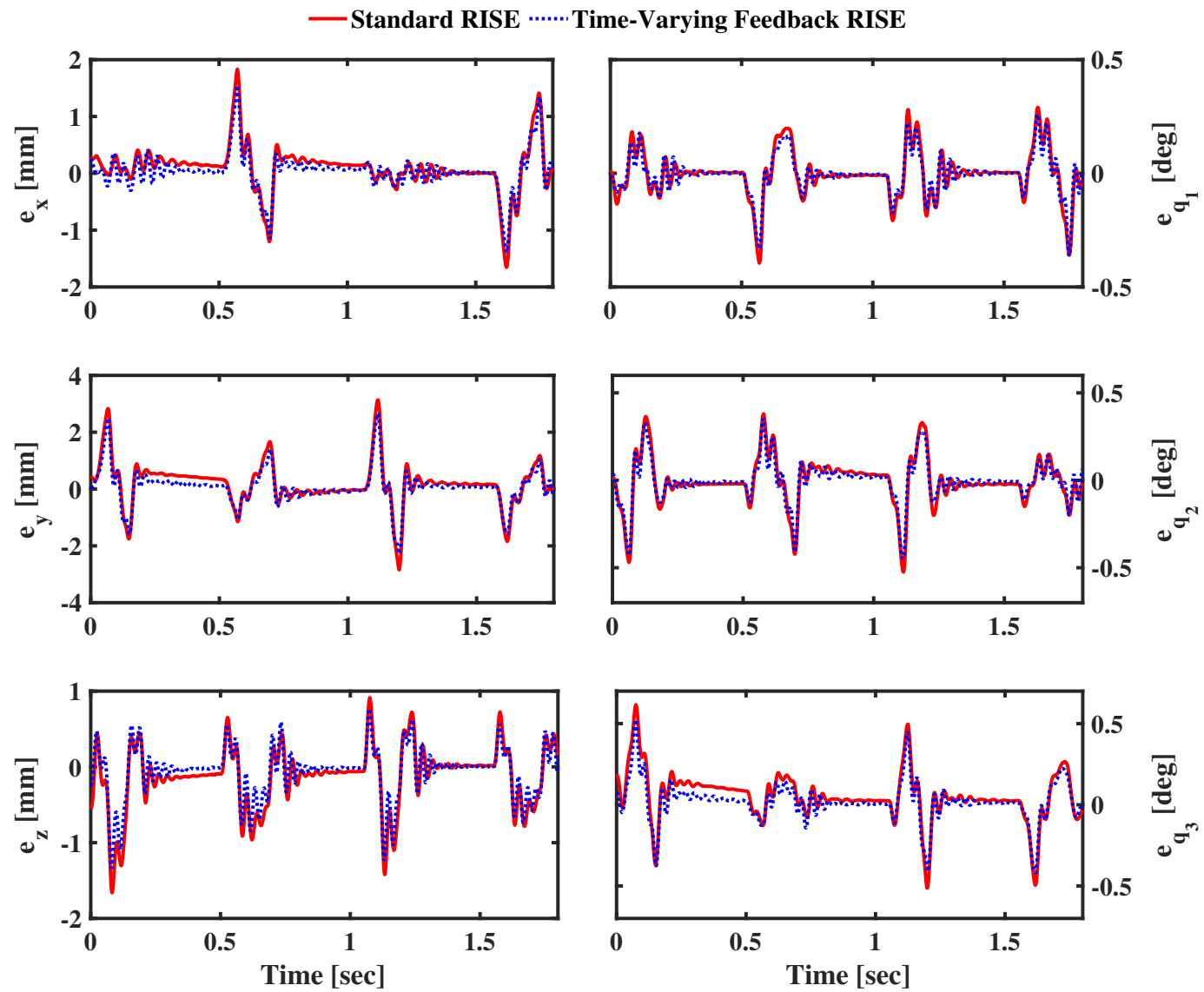

Figure 7. Scenario 1: Evolution of the Cartesian and joint tracking errors versus time. 

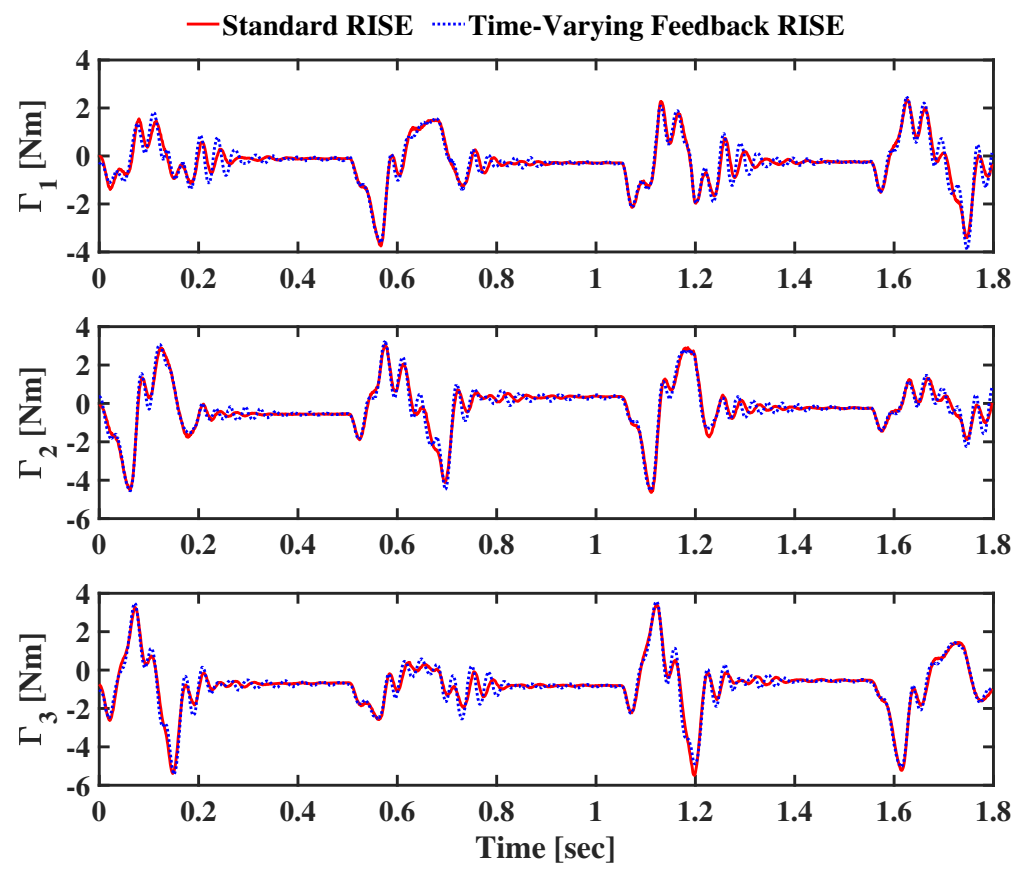

Figure 8. Scenario 1: Evolution of the control input torques versus time.

nonlinear feedback gains reduces the tracking errors in all axes. It can be seen that the peak errors are decreased and the general tracking errors of the proposed control are better than that of the standard RISE at different sections of the trajectory. One can quietly observe the better dynamic performance of the proposed control strategy compared to the original RISE control from the figured out tracking accuracy. The RMSE evaluated in both Cartesian and joint spaces and reported in Table 3, shows an improvement of $18 \%$ in terms of tracking precision.

The generated control input torques of the three direct-drive motors for both controllers are depicted in Figure 8. It is clear that both control algorithms generate an input signal within the admissible limits of the motors. No significant improvement is observed in terms of energy consumption in this scenario.

The evolution of the nonlinear feedback gains $\left(K_{s}()+1.\right)$ and $\left(k_{s 0}+1\right) \alpha_{2}($.$) overall$ the reference trajectory is depicted in Figures 9 and 10 respectively. The produced variations of both feedback gains versus time give always strictly positive bounded values (see Figures 9-a and 10-a). Figure 9-b shows the behavior of $\left(K_{s}()+1.\right)$ feedback gain versus the variation of the combined error $e_{2}$, which meets our expectations and desired performance. Moreover, Figure 10-b exposes the attitude of $\left(k_{s 0}+1\right) \alpha_{2}($.$) gain$ versus the variation of $\left(\int e_{2}\right)$ showing the performance in prospect, knowing that $\left(\int e_{2}\right)$ is always in the negative side.

Table 3. Scenario 1: Control perfromance evaluation for both controllers.

\begin{tabular}{ccc}
\hline & $R M S E_{x}[\mathrm{~mm}]$ & $R M S E_{J}[\mathrm{deg}]$ \\
\hline Original RISE & 0.993 & 0.2341 \\
New time-varying feedback RISE & 0.8129 & 0.192 \\
\hline Improvements & $18.1 \%$ & $18 \%$ \\
\hline
\end{tabular}



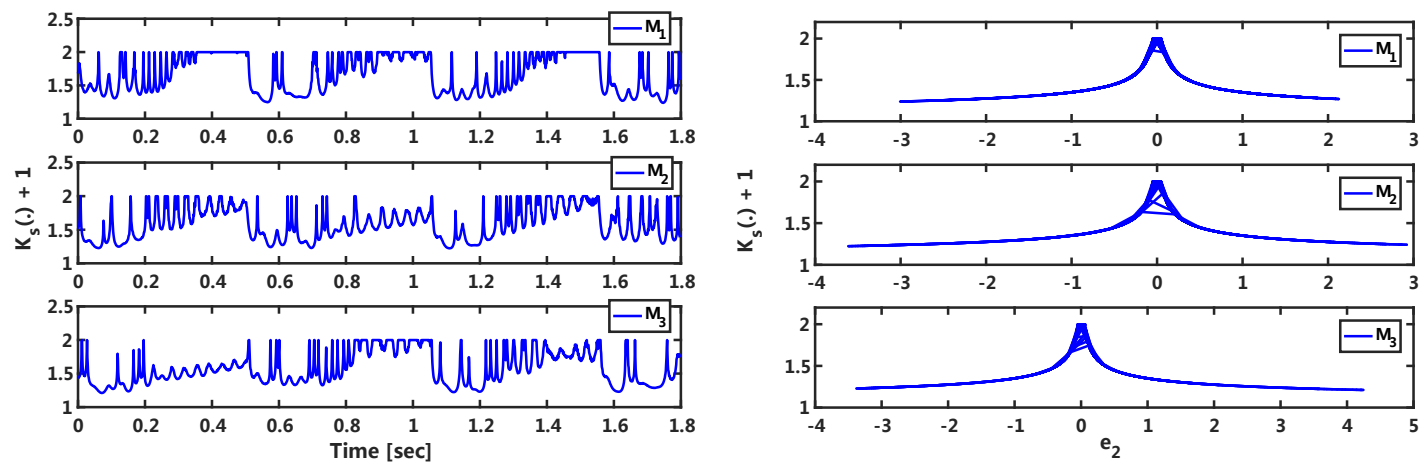

(a) $\left(K_{s}()+1.\right)$ versus time.

(b) $\left(K_{s}()+1.\right)$ versus combined error $e_{2}$.

Figure 9. Scenario 1: Evolution of the nonlinear feedback gain $\left(K_{s}()+1.\right)$.

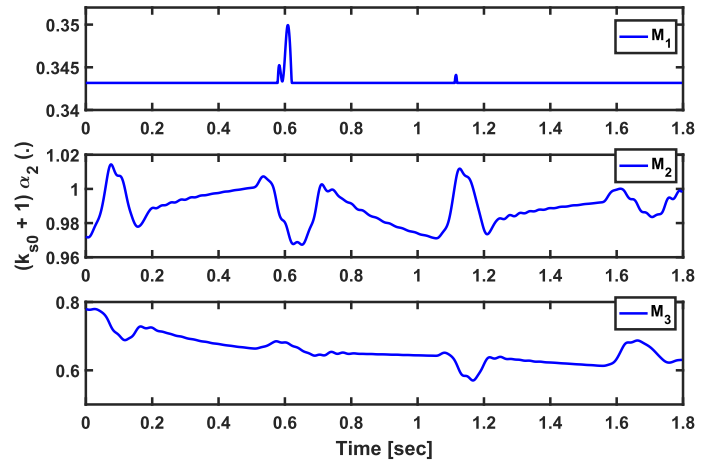

(a) $\left(k_{s 0}+1\right) \alpha_{2}($.$) versus time.$

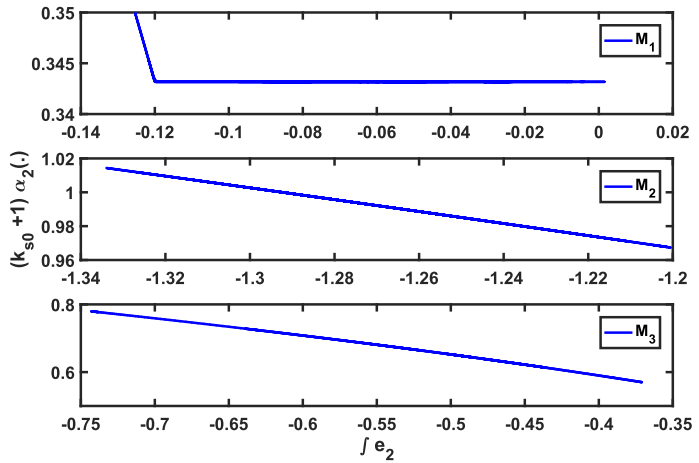

(b) $\left(k_{s 0}+1\right) \alpha_{2}($.$) versus \left(\int e_{2}\right)$.

Figure 10. Scenario 1: Evolution of the nonlinear feedback gain $\left(k_{s 0}+1\right) \alpha_{2}($.$) .$

This scenario shows clearly that the proposed time-varying feedback RISE control overcomes the original RISE control in terms of precision and performance thanks to the extended nonlinear feedback gains and their special behavior.

\subsection{Scenario 2: robustness towards speed and payload changes}

The main intended role of Delta parallel robot is performing rapid pick-and-place cycles for industrial applications such as food packaging. Accordingly, a payload of $225 \mathrm{~g}$ was attached to the traveling-plate of the robot accompanied by increasing the operating acceleration to $7.5 \mathrm{G}$ (with a speed of $2000 \mathrm{~mm} / \mathrm{s}$ ). The aim of this scenario is to test the robustness of the proposed time-varying feedback RISE controller towards payload and speed changes.

The same desired trajectory illustrated in Figures 5 and 6 is followed by the robot in this scenario. The resulted tracking errors in Cartesian and joint spaces for both controllers are displayed in Figure 11. Comparing to the original RISE tracking errors, a remarkable downsizing in the magnitudes of the tracking errors is obtained with the proposed time-varying feedback RISE controller. A significant improvement of $30.5 \%$ in terms of Cartesian space accuracy is noticed when evaluating the RMSE performance index and $28.3 \%$ for the joint space accuracy (see Table 4). Less oscillations are produced by the proposed time-varying feedback RISE control compared to the standard RISE control, especially in actuators 1 and 2 . 

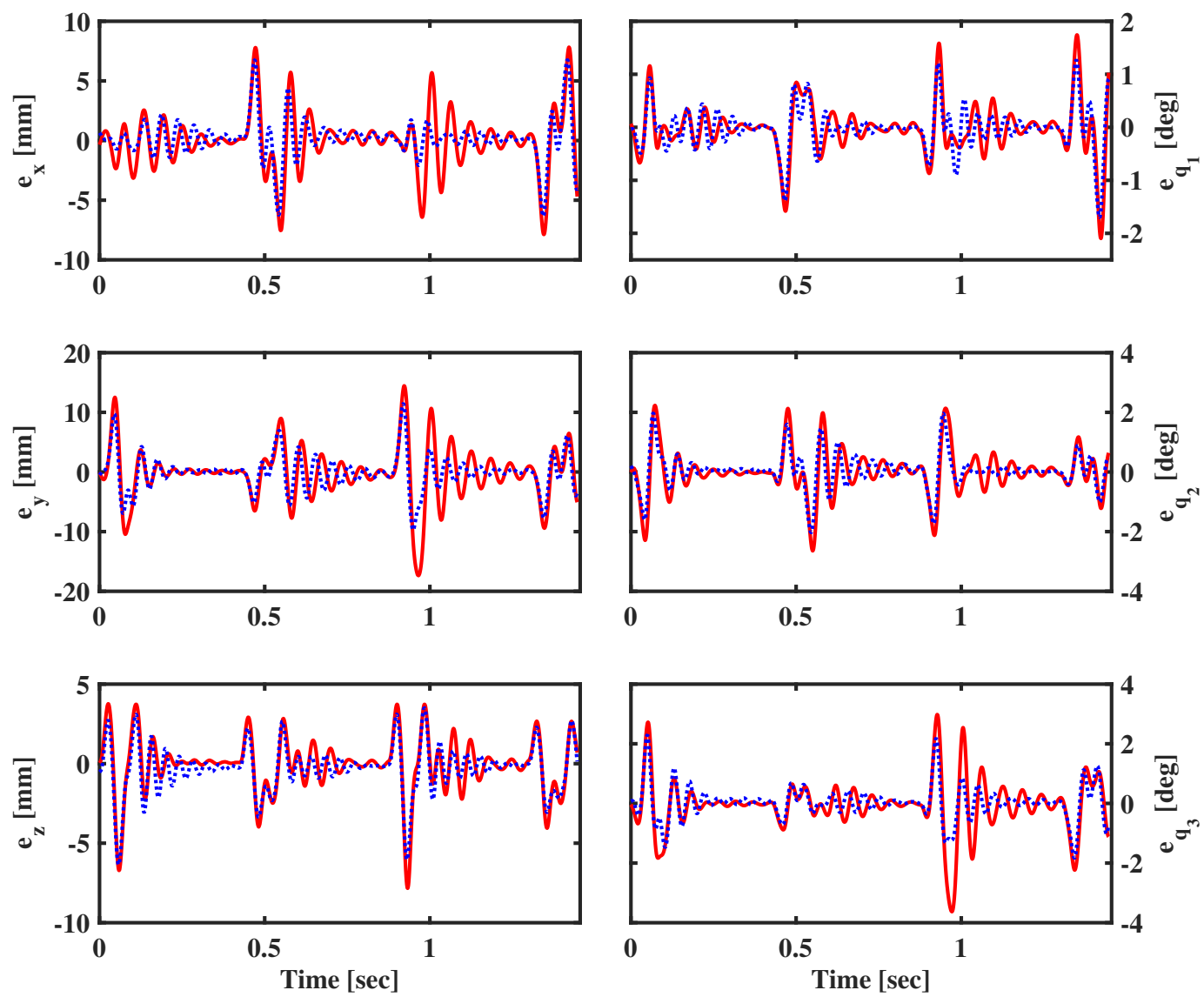

Figure 11. Scenario 2: Evolution of the Cartesian and joint tracking errors versus time.

Thus, the proposed control law is producing less tracking errors at high dynamic operating conditions (high-speed motions) with handled payload compared to the original RISE. This ensures the robustness of the proposed controller towards operating condition changes and parameters variation. Thanks to the nonlinear behavior of the time-varying feedback gains, the increased effect of the nonlinear dynamics of parallel manipulators at high-speed motions can be more compensated by the proposed control law in comparison to the original RISE even though no model-compensation term is considered.

Figure 12 displays the evolution of the control input torques along the reference trajectory for both controllers. Both standard RISE and proposed time-varying feedback RISE controllers guarantee an input signal within the safety margins of Delta robot actuators. Nevertheless, it is worth to highlight the reduced input torques generated by time-varying feedback RISE control compared to the standard RISE controller. In this scenario of high dynamic operating conditions, an improvement of $19.1 \%$ in terms of energy consumption is noticed and reported in Table 4 using the input-torques-based criterion proposed in (45).

The dynamic variations of the nonlinear feedback gains along the desired trajectory are depicted in Figures 13 and 14. It is obvious from Figures 13-a and 14-a to observe that the nonlinear gains remain positive and bounded even with the high changes of the dynamic operating conditions. Figure 13-b displays the action of $\left(K_{s}()+1.\right)$ gain 

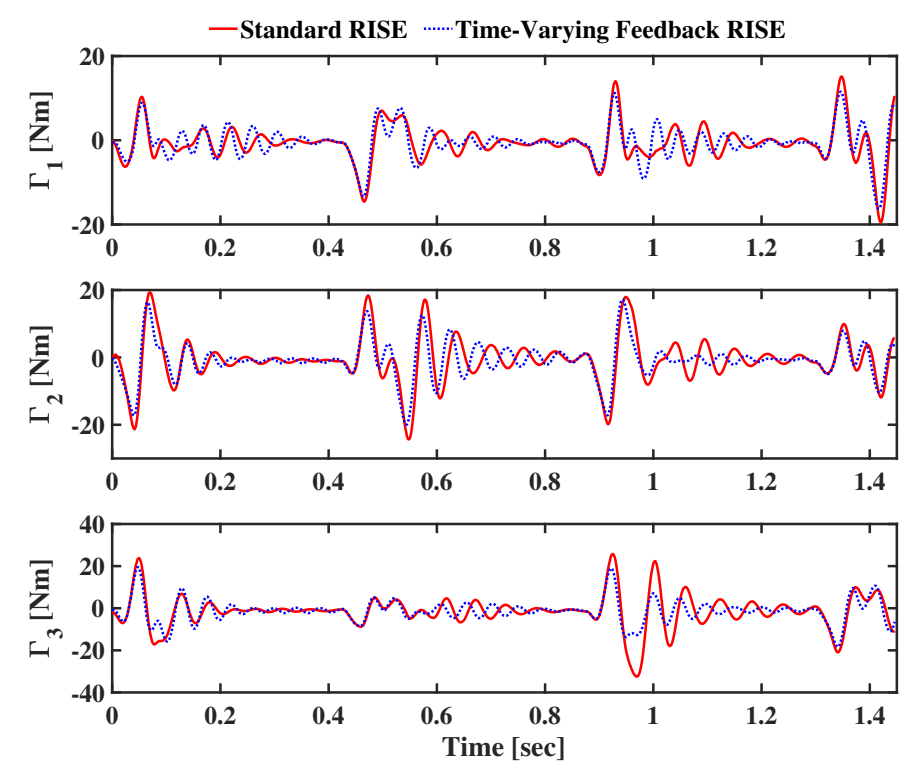

Figure 12. Scenario 2: Evolution of the control input torques versus time.

versus the combined error $e_{2}$ with a smaller constant zone compared to scenario 1 . This explains the better performance of the proposed time-varying feedback RISE controller compared to the original RISE controller by the dynamic behavior of the feedback gains with the change of the operating conditions. The same holds for $\left(k_{s 0}+1\right) \alpha_{2}($.$) gain in$ Figure 14-b, where the nonlinear variation is increased compared to scenario 1, leading to better control performance and more robustness.

To sum up, we can say that the proposed time-varying feedback RISE control is more robust towards payload and speed variations than the original RISE algorithm due to the dynamic behavior of the proposed nonlinear feedback gains.

\subsection{Performance index versus operating acceleration}

In this section, the operating acceleration of the robot is increased gradually starting from $2.5 \mathrm{G}$ reaching up $10 \mathrm{G}$. Both controllers have been tested at different operating acceleration with and without additional payload of $225 \mathrm{~g}$.

Figures 15 and 16 are two bar graphs showing the variation of the Cartesian RMSE in $(\mathrm{mm})$ with respect to the operating acceleration $(\mathrm{G})$ in case of no added payload and payload of $225 \mathrm{~g}$ respectively.

The improvement at each operating acceleration is evaluated for the two tested cases of payloads. It is shown in Figure 15 that in the case of no added payload, the improvement of the performance decreases as the acceleration increases. However, still the proposed time-varying feedback RISE controller performs better than the

Table 4. Scenario 2: Control perfromance evaluation for both controllers.

\begin{tabular}{cccc}
\hline & $R M S E_{x}[\mathrm{~mm}]$ & $R M S E_{J}[\mathrm{deg}]$ & $E_{\Gamma}[\mathrm{Nm}]$ \\
\hline Original RISE & 5.3985 & 1.2577 & $1.7692 \times 10^{4}$ \\
New time-varying feedback RISE & 3.7542 & 0.9012 & $1.4318 \times 10^{4}$ \\
\hline Improvements & $30.5 \%$ & $28.3 \%$ & $19.1 \%$ \\
\hline
\end{tabular}



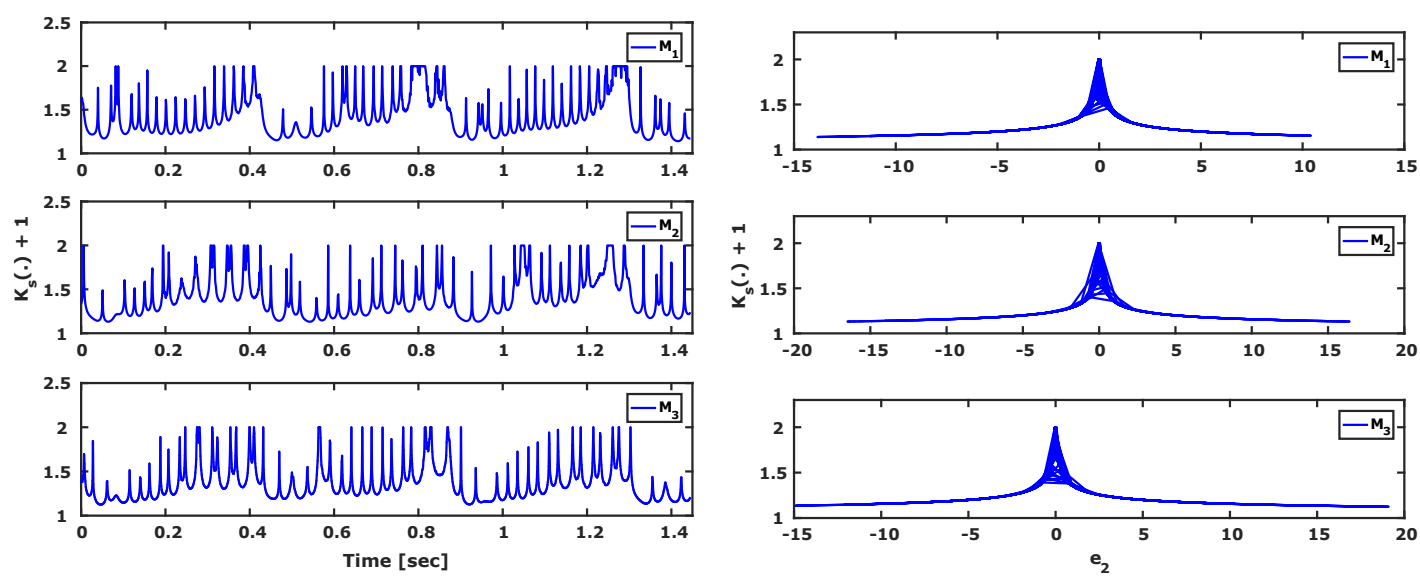

(a) $\left(K_{s}()+1.\right)$ versus time.

(b) $\left(K_{s}()+1.\right)$ versus combined error $e_{2}$.

Figure 13. Scenario 2: Evolution of the nonlinear feedback gain $\left(K_{s}()+1.\right)$.
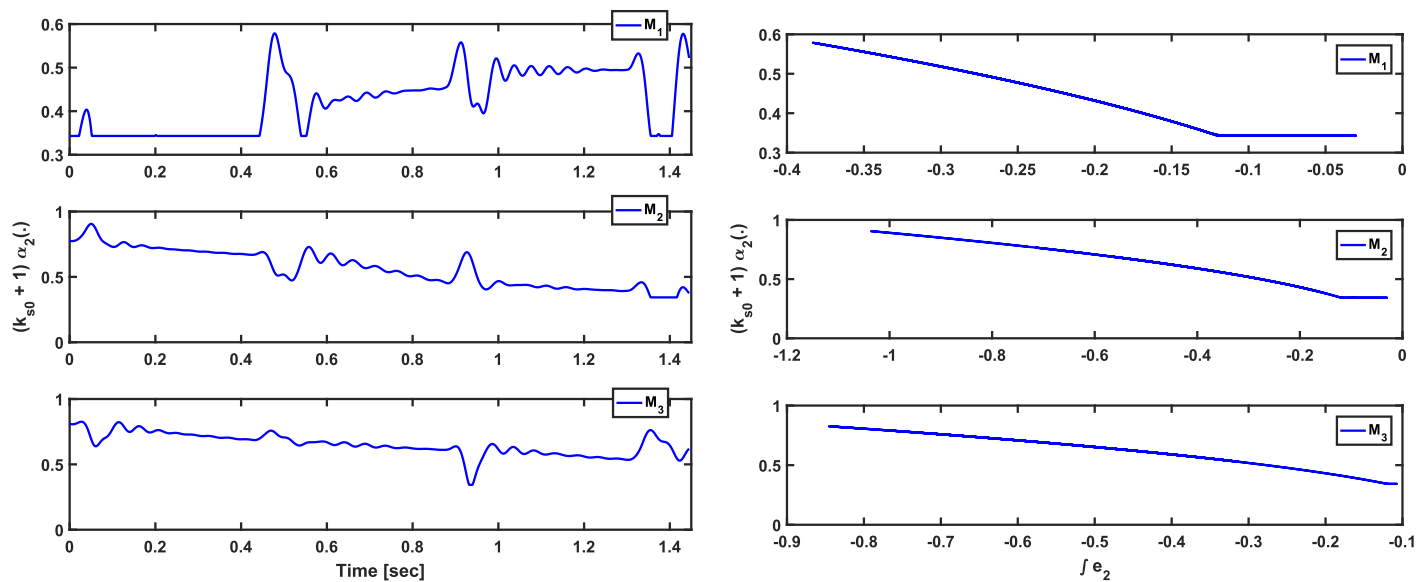

(a) $\left(k_{s 0}+1\right) \alpha_{2}($.$) versus time.$

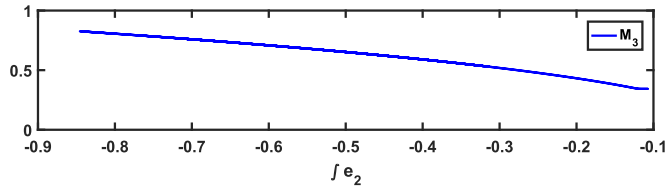

(b) $\left(k_{s 0}+1\right) \alpha_{2}($.$) versus \left(\int e_{2}\right)$.

Figure 14. Scenario 2: Evolution of the nonlinear feedback gain $\left(k_{s 0}+1\right) \alpha_{2}($.$) .$

original RISE at all operating accelerations. This proves the robustness of the proposed control strategy towards the effect of nonlinearities which increases considerably at high accelerations knowing that no dynamic compensator is used in the controller.

Furthermore, in the case of added payload of $225 \mathrm{~g}$, a significant improvement is observed at each operating acceleration as illustrated in Figure 16. In particular, at acceleration of $10 \mathrm{G}$, RISE controller generates high tracking errors overriding the safety margins of the robot. While the proposed time-varying feedback RISE controller generates acceptable tracking errors always within the defined safety margins. Thus, the robustness towards uncertainties and parameters variation at high dynamic operating conditions is verified by the proposed controller.

To this end, the experimental results have shown better performances of the proposed time-varying feedback RISE control strategy compared to the original RISE at different operating conditions. It has been verified that the proposed controller is more robust towards high dynamic operating condition changes (i.e. high-speed motions) with and without parameters variation (i.e. payload). 


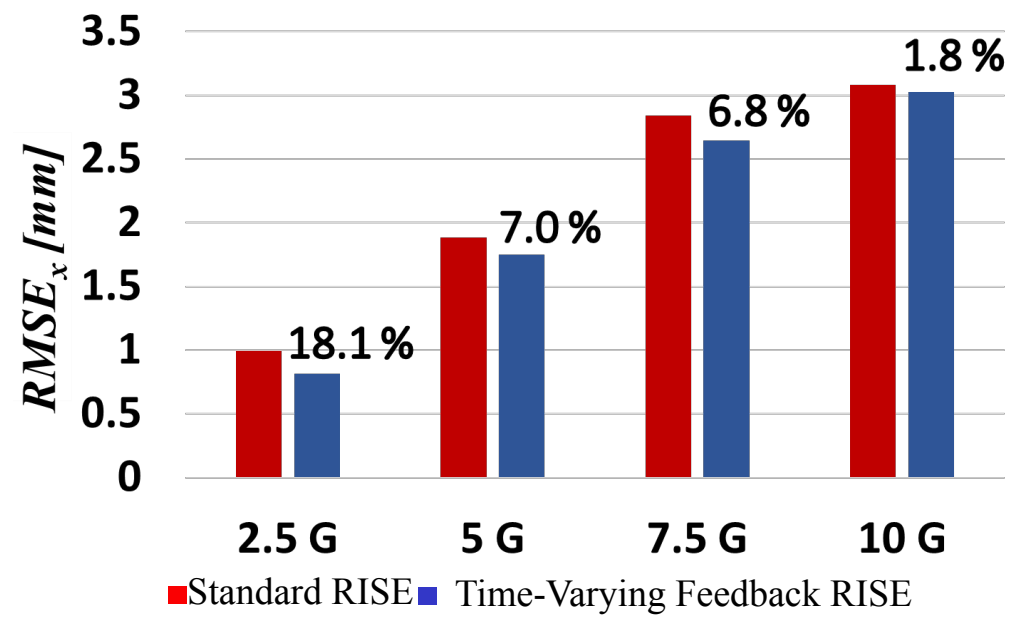

Figure 15. Clustered column chart of $R M S E_{x}$ versus operating acceleration with no payload.

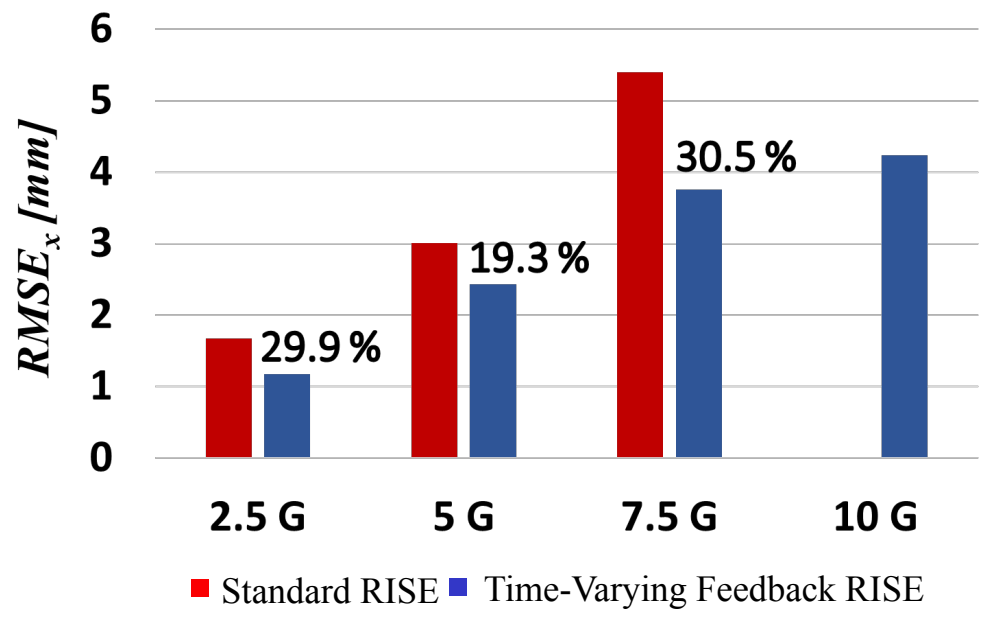

Figure 16. Clustered column chart of $R M S E_{x}$ versus operating acceleration with added payload of $225 \mathrm{~g}$.

\section{Conclusion and future work}

In this paper, a new class of RISE control strategy has been proposed for second-order nonlinear MIMO systems. RISE control law ensures semi-global asymptotic tracking under restricted assumptions on the uncertainties and nonlinearities of the system. We proposed to replace some of the static feedback gains in the original RISE control law by nonlinear ones depending on the system states. The idea is inspired by the fact that nonlinear time-varying gains lead mainly to better global performance and compensate for a wide range of nonlinearities and additive disturbances. A stability analysis has been established for the proposed controller. It proves that all the system signals are bounded and converge asymptotically to zero as time goes to infinity. Real-time experiments have been conducted on the 3-DOF non-redundant parallel robot Delta. The obtained experimental results show clearly better performance of the proposed time-varying feedback RISE -based control strategy compared to the original RISE controller in terms of tracking accuracy and robustness towards payload and speed changes. 
This work can be further extended by considering the dynamics of the system in adaptive algorithm with the proposed time-varying feedback RISE controller. A supplementary adaptive dynamic term rich enough with knowledge about the system may enhance the performance and robustness of such control law.

\section{References}

Bennehar, M., Chemori, A., Bouri, M., Jenni, L. F., \& Pierrot, F. (2017, February). A new RISE-based adaptive control of PKMs: design, stability analysis and experiments. International Journal of Control, 91(3), 593-607.

Bennehar, M., Chemori, A., \& Pierrot, F. (2016, July). A new revised desired compensation adaptive control for enhanced tracking: application to RA- PKMs. Advanced Robotics, 30(17-18), 1199-1214.

Bennehar, M., Chemori, A., Pierrot, F., Creuze, V. (2015, December). Extended Model-Based Feedforward Compensation in $\mathcal{L} 1$ Adaptive Control for Mechanical Manipulators: Design and Experiments. Frontiers in Robotics and AI, 2(32).

Bennehar, M., El Gazaly, G., Chemori, A., \& Pierrot, F. (2017, June). A novel adaptive terminal sliding mode control for parallel manipulators: Design and real-time experiments. In IEEE International Conference on Robotics and Automation (ICRA), Singapore, Singapore.

Clavel, R. (1990, December). Device for the movement and positioning of an element in space. Retrieved from https://patents.google.com/patent/US4976582A US Patent 4,976,582.

Codourey, A. (1996). Dynamic modelling and mass matrix evaluation of the DELTA parallel robot for axes decoupling control, Proceedings of IEEE/RSJ International Conference on Intelligent Robots and Systems (IROS'96). Osaka, Japan, 3, 1211-1218.

Codourey, A. (1998, December). Dynamic modeling of parallel robots for computed torque control implementation. The International Journal of Robotics Research, 17(12), 1325-1336.

Campos, E., Chemori, A., Creuze, V., Torres, J., \& Lozano, R. (2017, August). Saturation based nonlinear depth and yaw control of underwater vehicles with stability analysis and real-time experiments. Mechatronics, 45, 49-59.

Chaudhary, G., \& Ohri, J. (2016, July). 3-dof parallel manipulator control using pid controller. In IEEE 1st International Conference on Power Electronics, Intelligent Control and Energy Systems, Delhi, India.

Feemster, M. (2014, June). Jitter Reduction in a Directed Energy Application using RISE. In American Control Conference (ACC), Portland, Oregon, USA.

Fischer, N., Hughes, D., Walters, P., Schwartz, E. M., \& Dixon, W. E. (2014, August). Nonlinear RISE-Based Control of an Autonomous Underwater Vehicle. IEEE Transactions on Robotics, 30(4), 845-852.

Fischer, N., Kan, Z., Kamalapurkar, R., \& Dixon, W. (2014, April). Saturated RISE Feedback Control for a Class of Second-Order Nonlinear Systems. IEEE Transactions on Automatic Control, 59(4), 1094-1099.

Jingqing, H. (1994). Nonlinear PID controller. Acta Automatica Sinica, 20(4), 487-490.

Khalil, H. K. (1996-2002). Nonlinear Systems, 3rd ed. Prentice Hall, Upper Saddle River, New Jersey.

Kelly, R., \& Carelli, R. (1996, December). A class of nonlinear PDtype controllers for robot manipulators. Journal of Robotic Systems, 13(12), 793-802.

Kamaldin, N., Chen, S. L., Kong, C. G., Teo, C. S., \& Tan, K. K. (2016, July). Adaptive Parameter and Gain RISE Control of a Flexure-Based Dual-drive H Gantry. In IEEE International Conference on Advanced Intelligent Mechatronics (AIM), Alberta, Canada.

Kawai, Y., Namerikawa, T. (2013, August). Passivity and RISE based Robust Control for Bilateral Teleoperation System with Communication Delay. In IEEE International Conference on Control Applications (CCA), Hyderabad, India.

Luo, C., Yao, J., Chen, F., Li, L., \& Xu, Q. (2017, October). Adaptive Repetitive Control of 
Hydraulic Load Simulator With RISE Feedback. IEEE Access, Topic: Advanced modeling and control of complex mechatronic systems with nonlinearity and uncertainty, 5, 2390123911.

Merlet, J.-P. (2006). Parallel Robots, 2nd ed. Springer, Dordrecht, Netherlands.

Miller, K. \& Boris S. S. (1995). Modeling of Dynamics and Model-Based Control of DELTA Direct-Drive Parallel Robot. Journal of Robotics and Mechatronics, 7(4), 344-352.

Natal, G. S., Chemori, A. \& Pierrot, F. (2016, January). Nonlinear control of parallel manipulators for very high accelerations without velocity measurement: Stability analysis and experiments on par2 parallel manipulator. Robotica, 34(1), 43-70.

Natal, G. S., Chemori, A. \& Pierrot, F. (2015, July). Dual-space control of extremely fast parallel manipulators: Payload changes and the 100g experiment. IEEE Transactions on Control Systems Technology, 23(4), 1520-1535.

Patre, P. (2009). Lyapunov-based robust and adaptive control of nonlinear systems using a novel feedback structure. University of Florida.

Saied, H., Chemori, A., El Rafei, M., Francis, C., \& Pierrot, F. (2018, October). Actuator and Friction Dynamics Formulation in Control of PKMs: From Design to Real-Time Experiments. In IEEE/RSJ International Conference on Intelligent Robots and Systems (IROS'18), Madrid, Spain.

Shang, W. W., Cong, S., Li, Z. X., \& Jiang, S. L. (2009). Augmented Nonlinear PD Controller for a Redundantly Actuated Parallel Manipulator. Advanced Robotics, 23(12-13), 1725-1742.

Su, Y. X., Duan, B. Y., \& Zheng, C. H. (2004, January). Nonlinear PID Control of a Six-DOF Parallel Manipulator. IEE Proceedings - Control Theory and Applications, 151(1), 95-102.

Slotine, J.-J. E., \& Li, W. (1991). Applied Nonlinear Control. Prentice Hall, Englewood Cliffs, New Jersey.

Su, Y. X., Sun, D., \& Duan, B. Y. (2005, October). Design of an enhanced nonlinear PID controller. Mechatronics, 15(8), 1005-1024.

Xian, B., Dawson, D. M., Queiroz, M. S., \& Chen, J. (2004, July). A Continuous Asymptotic Tracking Control Strategy for Uncertain Nonlinear Systems. IEEE Transactions on Automatic control, 49(7), 1206-1211.

Xu, Y., Hollerbach, J. M., \& Ma, D. (1995, February). A Nonlinear PD Controller for Force and Contact Transient Control. IEEE Control Systems Magazine, 15(1), 15-21.

Yao, J., Deng, W., \& Jiao, Z. (2017, July). RISE-Based Adaptive Control of Hydraulic Systems With Asymptotic Tracking. IEEE Transactions on Automation Science and Engineering, $14(3), 1524-1531$.

Yao, J., Jiao, Z., \& Ma, D. (2014, December). RISE-Based Precision Motion Control of DC Motors With Continuous Friction Compensation. IEEE Transactions on Industrial Electronics, 61(12), 7067-7075. 